\title{
GLOBAL WELL-POSEDNESS OF THE ENERGY CRITICAL NONLINEAR SCHRÖDINGER EQUATION WITH SMALL INITIAL DATA IN $H^{1}\left(\mathbb{T}^{3}\right)$
}

\author{
SEBASTIAN HERR, DANIEL TATARU, AND NIKOLAY TZVETKOV
}

\begin{abstract}
A refined trilinear Strichartz estimate for solutions to the Schrödinger equation on the flat rational torus $\mathbb{T}^{3}$ is derived. By a suitable modification of critical function space theory this is applied to prove a small data global well-posedness result for the quintic Nonlinear Schrödinger Equation in $H^{s}\left(\mathbb{T}^{3}\right)$ for all $s \geq 1$. This is the first energy-critical global well-posedness result in the setting of compact manifolds.
\end{abstract}

\section{INTRODUCTION AND MAIN RESULT}

Our goal here is to establish a critical local well-posedness theory for the nonlinear Schrödinger equation

$$
\left(i \partial_{t}+\Delta\right) u=|u|^{4} u
$$

posed on the three dimensional torus $\mathbb{T}^{3}=\mathbb{R}^{3} /(2 \pi \mathbb{Z})^{3}$ and thus to naturally extend the result of Bourgain's fundamental paper [1] where only sub-critical regularity is considered. To our knowledge, this is the first critical well-posedness result in the case of a nonlinear Schrödinger equation on a compact manifold.

Since the critical space associated to (1) is the Sobolev space $H^{1}$, our result will directly imply small data global well-posedness for (1) (see the conservation of energy (5) below). The problem of arbitrary data global well-posedness is a remaining challenging issue.

If the problem (11) is posed on the Euclidean space $\mathbb{R}^{3}$ then one obtains small data global well-posedness by invoking the Strichartz estimate (see e.g. [11])

$$
\|u\|_{L_{t}^{\infty} H_{x}^{s}}+\|u\|_{L_{t}^{2} ; W_{x}^{s, 6}} \lesssim\|u(0)\|_{H^{s}}+\left\|\left(i \partial_{t}+\Delta\right) u\right\|_{L_{t}^{2} ; W_{x}^{s, 6 / 5}}
$$

Let us give the argument (see [7]). Applying (2) with $s=1$ in the context of (1) together with the Hölder and the Sobolev embedding

2000 Mathematics Subject Classification. 35Q55. 


$$
\begin{aligned}
& H^{1} \hookrightarrow L^{6} \text { yield } \\
& \qquad\|u\|_{L_{t}^{\infty} H_{x}^{1}}+\|u\|_{L_{t}^{2} ; W_{x}^{1,6}} \lesssim\|u(0)\|_{H^{1}}+\|u\|_{L_{t}^{2} W_{x}^{1,6}}\|u\|_{L_{t}^{\infty} H_{x}^{1}}^{4} .
\end{aligned}
$$

The above a priori estimate easily transforms to small data global wellposedness result for (11) by invoking the Picard iteration scheme (the use of the endpoint Strichartz estimate in this reasoning is not really needed). Recently, even the global well-posedness and scattering in the Euclidean setting has been established for large data by CollianderKeel-Staffilani-Takaoka-Tao [8].

The arguments of the Euclidean setting completely fail if (1) is posed on a compact Riemannian manifold. The Strichartz inequality (2) does not hold in the periodic case as one can see it by adapting to the $3 \mathrm{~d}$ situation the $1 \mathrm{~d}$ counterexample of [1]. It also strongly fails, if $\Delta$ is the Laplace-Beltrami operator on the three dimensional sphere by testing it for instance on zonal eigenfunctions with large eigenvalues (see [3]). The most dramatic failure is that of the non-homogeneous estimate.

As shown by the work of Bourgain [1], Burq-Gerard-Tzvetkov [3, 4, [5, 6] some weak versions of the inequality (2) survive in the setting of compact manifolds. For instance one has that for free solutions to the Schrödinger equation on $\mathbb{T}^{3}$, we have the estimate

$$
\|u\|_{L_{t}^{4} L_{x}^{4}} \lesssim\|u(0)\|_{H^{\frac{1}{4}+}} .
$$

In the Euclidean setting the same estimate without any loss is obtained from (2) combined with the Sobolev embedding $W^{\frac{1}{4}, 3}\left(\mathbb{R}^{3}\right) \hookrightarrow L^{4}\left(\mathbb{R}^{3}\right)$ (by interpolation one may also put $L_{t}^{4} L_{x}^{3}$ type norms in (2)). A similar estimate holds if the torus is replaced with the three dimensional sphere; there the epsilon loss can not be avoided, see [3].

Using the estimate (3) and its bilinear extensions one can prove essentially optimal (up to the critical regularity) well-posedness results for the nonlinear Schrödinger equation, on both the $3 d$ rational torus or the $3 d$ sphere (see [1], [5]). We also refer to [2] for partial results in the case of irrational tori.

In the present paper we shall be able to prove the endpoint result, i.e. well-posedness in the energy space $H^{1}$. For this purpose estimates with an epsilon loss of type (3) are useless. Our strategy is to use trilinear Strichartz type estimates on the right scales (with no loss with respect to scaling) only, involving $L_{t}^{p}$ with $p>2$, in the context of the $U^{p}$ and $V^{p}$ type critical spaces theory. These function spaces were originally developed in unpublished work on wave maps by the second author, see also Koch-Tataru [12]. Here we will use the formalism as presented in Hadac-Herr-Koch [9], but with the following refinement. The scaleinvariant linear Strichartz estimates which we aim to trilinearize involve 
GWP OF THE ENERGY CRITICAL NLS WITH SMALL DATA IN $H^{1}\left(\mathbb{T}^{3}\right)$

frequency scales which are finer than the standard dyadic localizations. Therefore, a modification of the norms is necessary which allows us to distinguish finer scales.

Let us now describe more precisely our results. Consider the Cauchy problem

$$
\begin{aligned}
i \partial_{t} u+\Delta u & =|u|^{4} u \\
u(0, x) & =\phi(x) \in H^{s}\left(\mathbb{T}^{3}\right) .
\end{aligned}
$$

For strong solutions $u$ of (4) we have energy conservation

$$
E(u(t))=\frac{1}{2} \int_{\mathbb{T}^{3}}|\nabla u(t, x)|^{2} d x+\frac{1}{6} \int_{\mathbb{T}^{3}}|u(t, x)|^{6} d x=E(\phi),
$$

and $L^{2}$-conservation

$$
M(u(t))=\frac{1}{2} \int_{\mathbb{T}^{3}}|u(t, x)|^{2} d x=M(\phi) .
$$

Thus, the natural energy space for this equation is $H^{1}\left(\mathbb{T}^{3}\right)$. The same problem (44) but considered in $\mathbb{R}^{3}$ is invariant with respect to the scaling law

$$
u(t, x) \rightarrow u_{\lambda}(t, x)=\lambda^{1 / 2} u\left(\lambda^{2} t, \lambda x\right)
$$

The energy is also invariant with respect to this scaling, which is why this problem is called energy critical. Scaling considerations remain valid in the periodic case for space-time scales which are $\ll 1$.

Define

$$
B_{\varepsilon}\left(\phi_{*}\right):=\left\{\phi \in H^{1}\left(\mathbb{T}^{3}\right):\left\|\phi-\phi_{*}\right\|_{H^{1}}<\varepsilon\right\} .
$$

Let us now state our first result dealing with local well-posedness.

Theorem 1.1 (Local well-posedness). Let $s \geq 1$. For every $\phi_{*} \in$ $H^{1}\left(\mathbb{T}^{3}\right)$ there exists $\varepsilon>0$ and $T=T\left(\phi_{*}\right)>0$ such that for all initial data $\phi \in B_{\varepsilon}\left(\phi_{*}\right) \cap H^{s}\left(\mathbb{T}^{3}\right)$ the Cauchy problem (4) has a unique solution

$$
u \in C\left([0, T) ; H^{s}\left(\mathbb{T}^{3}\right)\right) \cap X^{s}([0, T)) .
$$

This solution obeys the conservation laws (5) and (6), and the flow map

$$
B_{\varepsilon}\left(\phi_{*}\right) \cap H^{s}\left(\mathbb{T}^{3}\right) \ni \phi \mapsto u \in C\left([0, T) ; H^{s}\left(\mathbb{T}^{3}\right)\right) \cap X^{s}([0, T))
$$

is Lipschitz continuous.

The spaces $X^{s}$ involved in this statement are the critical spaces associated to our problem (see (11) below). In the case of small data, the proof of Theorem 1.1 shows that the life span $T$ of the solution only depends on the $H^{1}$-norm of the initial data. Thanks to the conservation laws, we therefore have a global in time result: 
Theorem 1.2 (Small data global well-posedness). Let $s \geq 1$. There exists $\varepsilon_{0}>0$ such that for all initial data $\phi \in B_{\varepsilon_{0}}(0) \cap H^{s}\left(\mathbb{T}^{3}\right)$ and every $T>0$ the Cauchy problem (4) has a unique solution

$$
u \in C\left([0, T) ; H^{s}\left(\mathbb{T}^{3}\right)\right) \cap X^{s}([0, T)) .
$$

This solution obeys the conservation laws (5) and (6), and the flow map

$$
B_{\varepsilon_{0}}(0) \cap H^{s}\left(\mathbb{T}^{3}\right) \ni \phi \mapsto u \in C\left([0, T) ; H^{s}\left(\mathbb{T}^{3}\right)\right) \cap X^{s}([0, T))
$$

is Lipschitz continuous.

The results of Theorem 1.1 and Theorem 1.2 hold equally well for the focusing problem

$$
i \partial_{t} u+\Delta u=-|u|^{4} u
$$

On the other hand in the large data global theory the difference between these two cases should of course be crucial.

At the moment we are not able to prove well-posedness in the energy space for (1) on the three dimensional sphere $\mathbb{S}^{3}$. Some progress in this direction was achieved in [6] where one gains control on the second Picard iteration in $H^{1}$. However the argument in [6] uses strongly separated interactions of the spatial and the time frequencies, a fact which does not fit well with the critical spaces machinery. In particular the control of the second Picard iteration performed in [6] does not rely only on $L_{t}^{p}, p>2$ properties of free solutions as is the case in the present paper.

The outline of the paper is as follows: In Section 2 we introduce some notation and the critical function spaces. In Section 3 we prove trilinear Strichartz type estimates which extend the results of J. Bourgain [1]. In Section 4 we show how these estimates imply our main results stated above.

We remark that for a proof of the crucial multilinear estimate in Proposition 4.1 the refinements involving localizations to rectangles in Section 3 are not necessary. Indeed, estimate (27) could be derived from the dyadic tri-linear Strichartz type estimate

$$
\left\|\prod_{j=1}^{3} P_{N_{j}} u_{j}\right\|_{L^{2}} \lesssim N_{2}^{1-\delta} N_{3}^{1+\delta} \prod_{j=1}^{3}\left\|P_{N_{j}} u_{j}\right\|_{Y^{0}}
$$

for some small $\delta>0$, which can be proved directly by orthogonality considerations, Hölder's inequality and the extension of Bourgain's estimate to critical function spaces (19). However, if one follows this strategy of proof, critical spaces which are sensible to finer than dyadic scales would still be needed. 
GWP OF THE ENERGY CRITICAL NLS WITH SMALL DATA IN $H^{1}\left(\mathbb{T}^{3}\right)$

We decided to present the stronger estimates because we believe that they are interesting in their own right, also for linear solutions. They may also prove useful in the study of the large data case. The technique we will use involves additional orthogonality considerations with respect to the temporal frequency of the solutions, not only with respect to the spatial frequencies. This circle of ideas has further applications, e.g. to $4 d$ cubic Schrödinger equations, see [10].

Acknowledgments. The authors are grateful to the anonymous referees for their valuable comments. In particular, a suggestion of one of the referees lead to a modification and simplification of Proposition 3.3, and as a consequence to an improvement of Corollary 3.4.

\section{CRitical FunCtion spaces}

Throughout this section let $H$ be a separable Hilbert space over $\mathbb{C}$. In our application this will be chosen as either $H^{s}\left(\mathbb{T}^{3}\right)$ or $\mathbb{C}$. We will briefly introduce the function spaces $U^{p}$ and $V^{p}$ (we refer the reader to [9. Section 2] for detailed proofs of the basic properties) and use them to construct all relevant function spaces for the present paper.

Let $\mathcal{Z}$ be the set of finite partitions $-\infty<t_{0}<t_{1}<\ldots<t_{K} \leq \infty$ of the real line. If $t_{K}=\infty$, we use the convention that $v\left(t_{K}\right):=0$ for all functions $v: \mathbb{R} \rightarrow H$. Let $\chi_{I}: \mathbb{R} \rightarrow \mathbb{R}$ denote the sharp characteristic function of a set $I \subset \mathbb{R}$.

Definition 2.1. Let $1 \leq p<\infty$. For $\left\{t_{k}\right\}_{k=0}^{K} \in \mathcal{Z}$ and $\left\{\phi_{k}\right\}_{k=0}^{K-1} \subset H$ with $\sum_{k=0}^{K-1}\left\|\phi_{k}\right\|_{H}^{p}=1$ we call the piecewise defined function $a: \mathbb{R} \rightarrow$ H,

$$
a=\sum_{k=1}^{K} \chi_{\left[t_{k-1}, t_{k}\right)} \phi_{k-1}
$$

a $U^{p}$-atom and we define the atomic space $U^{p}(\mathbb{R}, H)$ of all functions $u: \mathbb{R} \rightarrow H$ such that

$$
u=\sum_{j=1}^{\infty} \lambda_{j} a_{j} \text { for } U^{p} \text {-atoms } a_{j},\left\{\lambda_{j}\right\} \in \ell^{1},
$$

with norm

$$
\|u\|_{U^{p}}:=\inf \left\{\sum_{j=1}^{\infty}\left|\lambda_{j}\right|: u=\sum_{j=1}^{\infty} \lambda_{j} a_{j}, \lambda_{j} \in \mathbb{C}, a_{j} U^{p} \text {-atom }\right\} .
$$

Remark 1. The spaces $U^{p}(\mathbb{R}, H)$ are Banach spaces and we observe that $U^{p}(\mathbb{R}, H) \hookrightarrow L^{\infty}(\mathbb{R} ; H)$. Every $u \in U^{p}(\mathbb{R}, H)$ is right-continuous and $u$ tends to 0 for $t \rightarrow-\infty$. 
Definition 2.2. Let $1 \leq p<\infty$.

(i) We define $V^{p}(\mathbb{R}, H)$ as the space of all functions $v: \mathbb{R} \rightarrow H$ such that

$$
\|v\|_{V^{p}}:=\sup _{\left\{t_{k}\right\}_{k=0}^{K} \in \mathcal{Z}}\left(\sum_{k=1}^{K}\left\|v\left(t_{k}\right)-v\left(t_{k-1}\right)\right\|_{H}^{p}\right)^{\frac{1}{p}}
$$

is finite 1 .

(ii) Likewise, let $V_{r c}^{p}(\mathbb{R}, H)$ denote the closed subspace of all rightcontinuous functions $v: \mathbb{R} \rightarrow H$ such that $\lim _{t \rightarrow-\infty} v(t)=0$, endowed with the same norm (9).

Remark 2. The spaces $V^{p}(\mathbb{R}, H), V_{r c}^{p}(\mathbb{R}, H)$ are Banach spaces and satisfy $U^{p}(\mathbb{R}, H) \hookrightarrow V_{r c}^{p}(\mathbb{R}, H) \hookrightarrow L^{\infty}(\mathbb{R} ; H)$.

Proposition 2.3. For $1 \leq p<q<\infty$ we have $V_{r c}^{p}(\mathbb{R}, H) \hookrightarrow U^{q}(\mathbb{R}, H)$.

We also record a useful interpolation property of the spaces $U^{p}$ and $V^{p}$, cf. [9, Proposition 2.20].

Lemma 2.4. Let $q_{1}, q_{2}, q_{3}>2$, E be a Banach space and

$$
T: U^{q_{1}} \times U^{q_{2}} \times U^{q_{3}} \rightarrow E
$$

be a bounded, tri-linear operator with $\left\|T\left(u_{1}, u_{2}, u_{3}\right)\right\|_{E} \leq C \prod_{j=1}^{3}\left\|u_{j}\right\|_{U^{q_{j}}}$. In addition, assume that there exists $C_{2} \in(0, C]$ such that the estimate $\left\|T\left(u_{1}, u_{2}, u_{3}\right)\right\|_{E} \leq C_{2} \prod_{j=1}^{3}\left\|u_{j}\right\|_{U^{2}}$ holds true. Then, $T$ satisfies the estimate

$$
\left\|T\left(u_{1}, u_{2}, u_{3}\right)\right\|_{E} \lesssim C_{2}\left(\ln \frac{C}{C_{2}}+1\right)^{3} \prod_{j=1}^{3}\left\|u_{j}\right\|_{V^{2}}, \quad u_{j} \in V_{r c}^{2}, j=1,2,3 .
$$

Proof. For fixed $u_{2}, u_{3}$ let $T_{1} u:=T\left(u, u_{2}, u_{3}\right)$. From the assumption we have

$$
\begin{aligned}
& \left\|T_{1} u\right\|_{E} \leq D_{1}\|u\|_{U^{q_{1}}} \text { where } D_{1}=C\left\|u_{2}\right\|_{U^{q_{2}}}\left\|u_{3}\right\|_{U^{q_{3}}}, \\
& \left\|T_{1} u\right\|_{E} \leq D_{1}^{\prime}\|u\|_{U^{2}} \text { where } D_{1}^{\prime}=C_{2}\left\|u_{2}\right\|_{U^{2}}\left\|u_{3}\right\|_{U^{2}} .
\end{aligned}
$$

An application of [9, Proposition 2.20] and the bound $\left\|u_{j}\right\|_{U^{q_{j}}} \leq\left\|u_{j}\right\|_{U^{2}}$ for $j=2,3$ yield

$$
\left\|T_{1} u\right\|_{E} \lesssim C_{2}\left(\ln \frac{C}{C_{2}}+1\right)\|u\|_{V^{2}}\left\|u_{2}\right\|_{U^{2}}\left\|u_{3}\right\|_{U^{2}} .
$$

The embedding $V_{r c}^{2} \hookrightarrow U^{q_{j}}$ allows us to repeat this argument with respect to the second and third argument of $T$.

\footnotetext{
${ }^{1}$ Notice that here we use the convention $v(\infty)=0$
} 
We define the spatial Fourier coefficients

$$
\widehat{f}(\xi):=(2 \pi)^{-3 / 2} \int_{[0,2 \pi]^{3}} e^{-i x \cdot \xi} f(x) d x, \xi \in \mathbb{Z}^{3} .
$$

and the space time Fourier transform

$$
\mathcal{F} u(\tau, \xi):=(2 \pi)^{-2} \int_{\mathbb{R} \times[0,2 \pi]^{3}} e^{-i(x \cdot \xi+t \tau)} u(t, x) d t d x,(\tau, \xi) \in \mathbb{R} \times \mathbb{Z}^{3} .
$$

We fix a non-negative, even function $\psi \in C_{0}^{\infty}((-2,2))$ with $\psi(s)=1$ for $|s| \leq 1$ in order to define a partition of unity: for a dyadic number $N \geq 1$ we set

$$
\psi_{N}(\xi)=\psi\left(\frac{|\xi|}{N}\right)-\psi\left(\frac{2|\xi|}{N}\right), \quad \text { for } N \geq 2, \quad \psi_{1}(\xi)=\psi(|\xi|) .
$$

We define the frequency localization operators $P_{N}: L^{2}\left(\mathbb{T}^{3}\right) \rightarrow L^{2}\left(\mathbb{T}^{3}\right)$ as the Fourier multiplier with symbol $\psi_{N}$, and for brevity we also write $u_{N}:=P_{N} u$. Moreover, we define $P_{\leq N}:=\sum_{1 \leq M \leq N} P_{M}$.

More generally, for a set $S \subset \mathbb{Z}^{3}$ we define $P_{S}$ as the Fourier projection operator with symbol $\chi_{S}$, where $\chi_{S}$ denotes the characteristic function of $S$.

Let $s \in \mathbb{R}$. We define the Sobolev space $H^{s}\left(\mathbb{T}^{3}\right)$ as the space of all $L^{2}\left(\mathbb{T}^{3}\right)$-functions for which the norm

$$
\|f\|_{H^{s}\left(\mathbb{T}^{3}\right)}:=\left(\sum_{\xi \in \mathbb{Z}^{3}}\langle\xi\rangle^{2 s}|\hat{f}(\xi)|^{2}\right)^{\frac{1}{2}} \approx\left(\sum_{N \geq 1} N^{2 s}\left\|P_{N} f\right\|_{L^{2}\left(\mathbb{T}^{3}\right)}^{2}\right)^{\frac{1}{2}}
$$

is finite.

Corresponding to the linear Schrödinger flow we define

Definition 2.5. For $s \in \mathbb{R}$ we let $U_{\Delta}^{p} H^{s}$ resp. $V_{\Delta}^{p} H^{s}$ be the spaces of all functions $u: \mathbb{R} \rightarrow H^{s}\left(\mathbb{T}^{3}\right)$ such that $t \mapsto e^{-i t \Delta} u(t)$ is in $U^{p}\left(\mathbb{R}, H^{s}\right)$ resp. $V^{p}\left(\mathbb{R}, H^{s}\right)$, with norms

$$
\|u\|_{U_{\Delta}^{p} H^{s}}=\left\|e^{-i t \Delta} u\right\|_{U^{p}\left(\mathbb{R}, H^{s}\right)}, \quad\|u\|_{V_{\Delta}^{p} H^{s}}=\left\|e^{-i t \Delta} u\right\|_{V^{p}\left(\mathbb{R}, H^{s}\right)} .
$$

Spaces of this type have been succesfully used as replacements for $X^{s, b}$ spaces which are still effective at critical scaling, see for instance [12], [13, 9].

Remark 3. Proposition 2.3 and Lemma 2.4 naturally extend to the spaces $U_{\Delta}^{p} H^{s}$ and $V_{\Delta}^{p} H^{s}$.

In the context of this article this would suggest that spaces of the form $U_{\Delta}^{p} H^{1}$ and $V_{\Delta}^{p} H^{1}$ should be useful. However, here we introduce a small variation on this theme. 
Definition 2.6. For $s \in \mathbb{R}$ we define $X^{s}$ as the space of all functions $u: \mathbb{R} \rightarrow H^{s}\left(\mathbb{T}^{3}\right)$ such that for every $\xi \in \mathbb{Z}^{3}$ the map $t \mapsto e^{i t|\xi|^{2}} \widehat{u(t)}(\xi)$ is in $U^{2}(\mathbb{R}, \mathbb{C})$, and for which the norm

$$
\|u\|_{X^{s}}:=\left(\sum_{\xi \in \mathbb{Z}^{3}}\langle\xi\rangle^{2 s}\left\|e^{i t|\xi|^{2}} \widehat{u(t)}(\xi)\right\|_{U_{t}^{2}}^{2}\right)^{\frac{1}{2}}
$$

is finite.

Definition 2.7. Let $s \in \mathbb{R}$. We define $Y^{s}$ as the space of all functions $u: \mathbb{R} \rightarrow H^{s}\left(\mathbb{T}^{3}\right)$ such that for every $\xi \in \mathbb{Z}^{3}$ the map $t \mapsto e^{i t|\xi|^{2}} \widehat{u(t)}(\xi)$ is in $V_{r c}^{2}(\mathbb{R}, \mathbb{C})$, and for which the norm

$$
\|u\|_{Y^{s}}:=\left(\sum_{\xi \in \mathbb{Z}^{3}}\langle\xi\rangle^{2 s}\left\|e^{i t|\xi|^{2}} \widehat{u(t)}(\xi)\right\|_{V_{t}^{2}}^{2}\right)^{\frac{1}{2}}
$$

is finite.

As usual, for a time interval $I \subset \mathbb{R}$ we also consider the restriction spaces $X^{s}(I)$, etc. It is easy to relate the $X^{s}$ and $Y^{s}$ spaces with the previously defined $V_{\Delta}^{p} H^{s}$ and $U_{\Delta}^{p} H^{s}$. Using also Remark 2, we have:

Proposition 2.8. The following continuous embeddings hold:

$$
U_{\Delta}^{2} H^{s} \hookrightarrow X^{s} \hookrightarrow Y^{s} \hookrightarrow V_{\Delta}^{2} H^{s} .
$$

The motivation for the introduction of the $X^{s}$ and $Y^{s}$ spaces lies in the following

Corollary 2.9. Let $\mathbb{Z}^{3}=\cup C_{k}$ be a partition of $\mathbb{Z}^{3}$. Then

$$
\left(\sum_{k}\left\|P_{C_{k}} u\right\|_{V_{\Delta}^{2} H^{s}}^{2}\right)^{\frac{1}{2}} \lesssim\|u\|_{Y^{s}}
$$

The following Proposition follows immediately from the atomic structure of $U^{2}$.

Proposition 2.10. Let $s \geq 0,0<T \leq \infty$ and $\phi \in H^{s}\left(\mathbb{T}^{3}\right)$. Then, for the linear solution $u(t):=e^{i t \Delta} \phi$ for $t \geq 0$ we have $u \in X^{s}([0, T))$ and

$$
\|u\|_{X^{s}([0, T))} \leq\|\phi\|_{H^{s}}
$$

Let $f \in L_{l o c}^{1}\left([0, \infty) ; L^{2}\left(\mathbb{T}^{3}\right)\right)$ and define

$$
\mathcal{I}(f)(t):=\int_{0}^{t} e^{i(t-s) \Delta} f(s) d s
$$

for $t \geq 0$ and $\mathcal{I}(f)(t)=0$ otherwise. We have the following linear estimate for the Duhamel term. 
GWP OF THE ENERGY CRITICAL NLS WITH SMALL DATA IN $H^{1}\left(\mathbb{T}^{3}\right) \quad 9$

Proposition 2.11. Let $s \geq 0$ and $T>0$. For $f \in L^{1}\left([0, T) ; H^{s}\left(\mathbb{T}^{3}\right)\right)$ we have $\mathcal{I}(f) \in X^{s}([0, T))$ and

$$
\|\mathcal{I}(f)\|_{X^{s}([0, T))} \leq \sup _{v \in Y^{-s}([0, T)):\|v\|_{Y^{-s}}=1}\left|\int_{0}^{T} \int_{\mathbb{T}^{3}} f(t, x) \overline{v(t, x)} d x d t\right|
$$

Proof. We extend $\mathcal{I}(f)$ continuously by $\mathcal{I}(f)(t):=\int_{0}^{T} e^{i(t-s) \Delta} f(s) d s$ for $t>T$. For each $\xi$ we have that $t \rightarrow e^{i t|\xi|^{2}} \widehat{\mathcal{I}(f)(t)}(\xi)$ is absolutely continuous and of bounded variation, hence in $U^{2}$. Let $\varepsilon>0$. There is a sequence $\left(b_{\xi}\right)_{\xi \in \mathbb{Z}^{3}} \in \ell^{2}\left(\mathbb{Z}^{3}\right),\left\|\left(b_{\xi}\right)\right\|_{\ell^{2}}=1$ such that

$$
\|\mathcal{I}(f)\|_{X^{s}([0, T))} \leq \sum_{\xi \in \mathbb{Z}^{3}} b_{\xi}\langle\xi\rangle^{s}\left\|\int_{0}^{t} \chi_{[0, T)}(s) e^{i s|\xi|^{2}} \widehat{f(s)}(\xi) d s\right\|_{U_{t}^{2}}+\varepsilon .
$$

By duality of $U^{2}$ and $V^{2}$, see [9, Theorem 2.8 and Proposition 2.10], for each $\xi \in \mathbb{Z}^{3}$ there exists $v_{\xi} \in V_{t}^{2},\left\|v_{\xi}\right\|_{V^{2}}=1$, such that

$$
\left\|\int_{0}^{t} \chi_{[0, T)}(s) e^{i s|\xi|^{2}} \widehat{f(s)}(\xi) d s\right\|_{U_{t}^{2}} \leq\left|\int_{0}^{T} \widehat{f(s)}(\xi) \overline{e^{-i s|\xi|^{2}} v_{\xi}(s)} d s\right|+2^{-|\xi|^{2}} \varepsilon .
$$

Evidently, we can choose $v_{\xi}$ to be right-continuous with supp $v_{\xi} \subseteq[0, T)$ without changing the above expression. Define

$$
v(t, x)=(2 \pi)^{-3 / 2} \sum_{\xi \in \mathbb{Z}^{3}} b_{\xi}\langle\xi\rangle^{s} e^{i x \cdot \xi} e^{-i t|\xi|^{2}} v_{\xi}(t) .
$$

Then, $v \in Y^{-s}([0, T)),\|v\|_{Y^{-s}} \leq 1$, and

$$
\|\mathcal{I}(f)\|_{X^{s}([0, T))} \leq \sum_{\xi \in \mathbb{Z}^{3}}\left|\int_{0}^{T} \widehat{f(t)}(\xi) \overline{\widehat{v(t)(\xi)}} d t\right|+c \varepsilon
$$

and the claim follows by dominated convergence and Plancherel's identity.

Remark 4. In particular, Proposition 2.11 implies that any estimate on $f$ in $L^{1}\left([0, T) ; H^{s}\left(\mathbb{T}^{3}\right)\right)$ bounds $\mathcal{I}(f)$ in $X^{s}([0, T))$.

\section{Strichartz estimates}

We first recall Bourgain's $L^{p}$ estimates of Strichartz type. Let $\mathcal{C}_{N}$ denote the collection of cubes $C \subset \mathbb{Z}^{3}$ of side-length $N \geq 1$ with arbitrary center and orientation.

Proposition 3.1 (Bourgain [1]). Let $p>4$. For all $N \geq 1$ we have

$$
\left\|P_{N} e^{i t \Delta} \phi\right\|_{L^{p}\left(\mathbb{T} \times \mathbb{T}^{3}\right)} \lesssim N^{\frac{3}{2}-\frac{5}{p}}\left\|P_{N} \phi\right\|_{L^{2}\left(\mathbb{T}^{3}\right)} .
$$


More generally, for all $C \in \mathcal{C}_{N}$ we have

$$
\left\|P_{C} e^{i t \Delta} \phi\right\|_{L^{p}\left(\mathbb{T} \times \mathbb{T}^{3}\right)} \lesssim N^{\frac{3}{2}-\frac{5}{p}}\left\|P_{C} \phi\right\|_{L^{2}\left(\mathbb{T}^{3}\right)} .
$$

Proof/Reference. Estimate (17) follows [1, formula 3.117]. Let $C \in \mathcal{C}_{N}$ with center $\xi_{0}$. We first apply a Galilean transformation (as in [1, formulas 5.7-5.8]) to shift the center of the cube $C$ to the origin and replace $C$ by $C_{0}=C-\xi_{0}$. Denoting $\phi_{0}(x)=e^{-i x \cdot \xi_{0}} \phi(x)$ we have $\widehat{\phi}_{0}(\xi)=\widehat{\phi}\left(\xi+\xi_{0}\right)$ and therefore $\left\|P_{C} \phi\right\|_{L^{2}\left(\mathbb{T}^{3}\right)}=\left\|P_{C_{0}} \phi_{0}\right\|_{L^{2}\left(\mathbb{T}^{3}\right)}$. Furthermore, we can relate the corresponding solutions to the Schrödinger equations. We have

$$
P_{C_{0}} e^{i t \Delta} \phi_{0}(t, x)=\sum_{\xi \in C_{0}} e^{i\left(x \cdot \xi-t|\xi|^{2}\right)} \widehat{\phi}_{0}(\xi)=\sum_{\xi \in C} e^{i\left(x \cdot\left(\xi-\xi_{0}\right)-t\left|\xi-\xi_{0}\right|^{2}\right)} \widehat{\phi}(\xi)
$$

therefore by rewriting the phase

$$
x \cdot\left(\xi-\xi_{0}\right)-t\left|\xi-\xi_{0}\right|^{2}=\left(x+2 t \xi_{0}\right) \cdot \xi-t|\xi|^{2}-x \cdot \xi_{0}-t\left|\xi_{0}\right|^{2}
$$

we obtain

$$
P_{C_{0}} e^{i t \Delta} \phi_{0}(t, x)=e^{-i\left(x \xi_{0}+t\left|\xi_{0}\right|^{2}\right)} P_{C} e^{i t \Delta} \phi\left(t, x+2 t \xi_{0}\right)
$$

Thus $\left\|P_{C} e^{i t \Delta} \phi\right\|_{L^{p}\left(\mathbb{T} \times \mathbb{T}^{3}\right)}=\left\|P_{C_{0}} e^{i t \Delta} \phi_{0}\right\|_{L^{p}\left(\mathbb{T} \times \mathbb{T}^{3}\right)}$. The bound (18) with $C$ replaced by $C_{0}$ follows from (17).

Corollary 3.2. Let $p>4$. For all $N \geq 1$ and $C \in \mathcal{C}_{N}$ we have

$$
\left\|P_{C} u\right\|_{L^{p}\left(\mathbb{T} \times \mathbb{T}^{3}\right)} \lesssim N^{\frac{3}{2}-\frac{5}{p}}\left\|P_{C} u\right\|_{U_{\Delta}^{p} L^{2}} .
$$

Proof. Due to the atomic structure of $U^{p}$ it suffices to consider a piecewise linear solution $a$, i.e.

$$
a(t)=\sum_{k=1}^{K} \chi_{\left[t_{k-1}, t_{k}\right)}(t) e^{i t \Delta} \phi_{k-1} \text { with } \sum_{k=0}^{K}\left\|\phi_{k-1}\right\|_{L^{2}}^{p}=1
$$

We apply (18) on each interval $\left[t_{k-1}, t_{k}\right)$, and then sum up with respect to $k$.

In the remainder of this section we will refine Bourgain's estimate (17) in the case $p>4$ by proving stronger bounds for solutions which are frequency-localized to $3 d$ rectangles.

Let $\mathcal{R}_{M}(N)$ be the collection of all sets in $\mathbb{Z}^{3}$ which are given as the intersection of a cube of sidelength $2 N$ with strips of width $2 M$, i.e. the collection of all sets of the form

$$
\left(\xi_{0}+[-N, N]^{3}\right) \cap\left\{\xi \in \mathbb{Z}^{3}:|a \cdot \xi-A| \leq M\right\}
$$

with some $\xi_{0} \in \mathbb{Z}^{3}, a \in \mathbb{R}^{3},|a|=1, A \in \mathbb{R}$. 
GWP OF THE ENERGY CRITICAL NLS WITH SMALL DATA IN $H^{1}\left(\mathbb{T}^{3}\right)$

11

Proposition 3.3. For all $1 \leq M \leq N$ and $R \in \mathcal{R}_{M}(N)$ we have

$$
\left\|P_{R} e^{i t \Delta} \phi\right\|_{L^{\infty}\left(\mathbb{T} \times \mathbb{T}^{3}\right)} \lesssim M^{\frac{1}{2}} N\left\|P_{R} \phi\right\|_{L^{2}\left(\mathbb{T}^{3}\right)} .
$$

Proof. For fixed $t$ we have the uniform bound

$$
\left\|P_{R} e^{i t \Delta} \phi\right\|_{L^{\infty}\left(\mathbb{T}^{3}\right)} \leq \sum_{\xi \in \mathbb{Z}^{3} \cap R}|\widehat{\phi}(\xi)| \leq\left(\#\left(\mathbb{Z}^{3} \cap R\right)\right)^{\frac{1}{2}}\left\|P_{R} \phi\right\|_{L^{2}\left(\mathbb{T}^{3}\right)} .
$$

We cover the rectangle by $\sim N^{2} / M^{2}$ cubes of side-length $M$ and faces parallel to the coordinate planes. Each such cube contains $\sim M^{3}$ lattice points. Altogether, we arrive at the upper bound

$$
\#\left(\mathbb{Z}^{3} \cap R\right) \lesssim M N^{2}
$$

which shows the claim.

The estimates (18) and (20) and Hölder's inequality imply

Corollary 3.4. Let $p>4$ and $0<\delta<\frac{1}{2}-\frac{2}{p}$. For all $1 \leq M \leq N$ and $R \in \mathcal{R}_{M}(N)$ we have

$$
\left\|P_{R} e^{i t \Delta} \phi\right\|_{L^{p}\left(\mathbb{T} \times \mathbb{T}^{3}\right)} \lesssim N^{\frac{3}{2}-\frac{5}{p}}\left(\frac{M}{N}\right)^{\delta}\left\|P_{R} \phi\right\|_{L^{2}\left(\mathbb{T}^{3}\right)} .
$$

We conclude the section with our key trilinear estimate:

Proposition 3.5. There exists $\delta>0$ such that for any $N_{1} \geq N_{2} \geq$ $N_{3} \geq 1$ and any interval $I \subset[0,2 \pi]$ we have

$$
\left\|\prod_{j=1}^{3} P_{N_{j}} u_{j}\right\|_{L^{2}\left(I \times \mathbb{T}^{3}\right)} \lesssim N_{2} N_{3} \max \left\{\frac{N_{3}}{N_{1}}, \frac{1}{N_{2}}\right\}^{\delta} \prod_{j=1}^{3}\left\|P_{N_{j}} u_{j}\right\|_{Y^{0}} .
$$

Proof. We begin the proof with some simplifications. First of all, it is enough to consider the case $I=[0,2 \pi]$. Second, given a partition $\mathbb{Z}^{3}=\cup C_{j}$ into cubes $C_{j} \in \mathcal{C}_{N_{2}}$, the outputs $P_{N_{1}} P_{C_{j}} u_{1} P_{N_{2}} u_{2} P_{N_{3}} u_{3}$ are almost orthogonal because the spatial Fourier-support of $u_{2} P_{N_{3}} u_{3}$ is contained in at most finitely many cubes of sidelength $N_{2}$. Additionally, we have

$$
\|u\|_{Y^{0}}^{2}=\sum_{j}\left\|P_{C_{j}} u\right\|_{Y^{0}}^{2},
$$

which implies that it suffices to prove (22) in the case when the first factor is further restricted to a cube $C \in \mathcal{C}_{N_{2}}$,

$$
\left\|P_{N_{1}} P_{C} u_{1} P_{N_{2}} u_{2} P_{N_{3}} u_{3}\right\|_{L^{2}} \lesssim N_{2} N_{3}\left(\frac{N_{3}}{N_{1}}+\frac{1}{N_{2}}\right)^{\delta} \prod_{j=1}^{3}\left\|P_{N_{j}} u_{j}\right\|_{Y^{0}}
$$

where $L^{2}=L^{2}\left([0,2 \pi] \times \mathbb{T}^{3}\right)$. 
In (23), thanks to Proposition [2.8, we are allowed to replace $Y^{0}$ by $V_{\Delta}^{2} L^{2}$. Then (23) follows by interpolation, via our interpolation Lemma 2.4, from the following two trilinear estimates:

$$
\left\|P_{C} P_{N_{1}} u_{1} P_{N_{2}} u_{2} P_{N_{3}} u_{3}\right\|_{L^{2}} \lesssim N_{2} N_{3}\left(\frac{N_{3}}{N_{2}}\right)^{\delta^{\prime}} \prod_{j=1}^{3}\left\|P_{N_{j}} u_{j}\right\|_{U_{\Delta}^{4} L^{2}}
$$

for $0<\delta^{\prime}<\frac{1}{2}$, respectively

$$
\left\|P_{C} P_{N_{1}} u_{1} P_{N_{2}} u_{2} P_{N_{3}} u_{3}\right\|_{L^{2}} \lesssim N_{2} N_{3}\left(\frac{N_{3}}{N_{1}}+\frac{1}{N_{2}}\right)^{\delta^{\prime \prime}} \prod_{j=1}^{3}\left\|P_{N_{j}} u_{j}\right\|_{U_{\Delta}^{2} L^{2}}
$$

for some $\delta^{\prime \prime}>0$.

The first bound (24) follows from Hölder's inequality with $4<p<5$ and $q$ such that $2 / p+1 / q=1 / 2$ and (19)

$$
\begin{aligned}
& \left\|P_{C} P_{N_{1}} u_{1} P_{N_{2}} u_{2} P_{N_{3}} u_{3}\right\|_{L^{2}} \leq\left\|P_{C} P_{N_{1}} u_{1}\right\|_{L^{p}}\left\|P_{N_{2}} u_{2}\right\|_{L^{p}}\left\|P_{N_{3}} u_{3}\right\|_{L^{q}} \\
\lesssim & N_{2}^{3-\frac{10}{p}} N_{3}^{\frac{3}{2}-\frac{5}{q}}\left\|P_{N_{1}} u_{1}\right\|_{U_{\Delta}^{p} L^{2}}\left\|P_{N_{2}} u_{2}\right\|_{U_{\Delta}^{p} L^{2}}\left\|P_{N_{3}} u_{3}\right\|_{U_{\Delta}^{q} L^{2}},
\end{aligned}
$$

and the embedding $U_{\Delta}^{4} L^{2} \hookrightarrow U_{\Delta}^{p} L^{2} \hookrightarrow U_{\Delta}^{q} L^{2}$.

For the second bound (25) we can use the atomic structure of the $U^{2}$ spaces (see e.g. [9, Proposition 2.19] for more details on this point) to reduce the problem to the similar estimate for the product of three solutions to the linear Schrödinger equation $u_{j}=e^{i t \Delta} \phi_{j}$ :

$$
\left\|P_{C} P_{N_{1}} u_{1} P_{N_{2}} u_{2} P_{N_{3}} u_{3}\right\|_{L^{2}} \lesssim N_{2} N_{3}\left(\frac{N_{3}}{N_{1}}+\frac{1}{N_{2}}\right)^{\delta^{\prime \prime}} \prod_{j=1}^{3}\left\|P_{N_{j}} \phi_{j}\right\|_{L^{2}} .
$$

Let $\xi_{0}$ be the center of $C$. We partition $C=\cup R_{k}$ into almost disjoint strips of width $M=\max \left\{N_{2}^{2} / N_{1}, 1\right\}$ which are orthogonal to $\xi_{0}$,

$$
R_{k}=\left\{\xi \in C: \xi \cdot \xi_{0} \in\left[\left|\xi_{0}\right| M k,\left|\xi_{0}\right| M(k+1)\right)\right\}, \quad|k| \approx N_{1} / M
$$

Each $R_{k}$ is the intersection of a cube of sidelegth $2 N_{2}$ with a strip of width $M$, and we decompose

$$
P_{C} P_{N_{1}} u_{1} P_{N_{2}} u_{2} P_{N_{3}} u_{3}=\sum_{k} P_{R_{k}} P_{N_{1}} u_{1} P_{N_{2}} u_{2} P_{N_{3}} u_{3}
$$

and claim that the summands are almost orthogonal in $L^{2}\left(\mathbb{T} \times \mathbb{T}^{3}\right)$. This orthogonality no longer comes from the spatial frequencies, but from the time frequency. Indeed, for $\xi_{1} \in R_{k}$ we have

$$
\xi_{1}^{2}=\frac{1}{\left|\xi_{0}\right|^{2}}\left|\xi_{1} \cdot \xi_{0}\right|^{2}+\left|\xi_{1}-\xi_{0}\right|^{2}-\frac{1}{\left|\xi_{0}\right|^{2}}\left|\left(\xi_{1}-\xi_{0}\right) \cdot \xi_{0}\right|^{2}=M^{2} k^{2}+O\left(M^{2} k\right)
$$


since $N_{2}^{2} \lesssim M^{2} k$. The second and third factor alter the time frequency by at most $O\left(N_{2}^{2}\right)$. Hence the expressions $P_{R_{k}} P_{N_{1}} u_{1} P_{N_{2}} u_{2} P_{N_{3}} u_{3}$ are localized at time frequency $M^{2} k^{2}+O\left(M^{2} k\right)$ and thus are almost orthogonal,

$$
\left\|P_{C} P_{N_{1}} u_{1} P_{N_{2}} u_{2} P_{N_{3}} u_{3}\right\|_{L^{2}}^{2} \lesssim \sum_{k}\left\|P_{R_{k}} P_{N_{1}} u_{1} P_{N_{2}} u_{2} P_{N_{3}} u_{3}\right\|_{L^{2}}^{2}
$$

On the other hand the estimates (21) and (18) yield

$$
\begin{aligned}
\left\|P_{R_{k}} P_{N_{1}} u_{1} P_{N_{2}} u_{2} P_{N_{3}} u_{3}\right\|_{L^{2}} \lesssim & N_{2}^{3-\frac{10}{p}}\left(\frac{M}{N_{2}}\right)^{\varepsilon} N_{3}^{\frac{3}{2}-\frac{5}{q}} \\
& \left\|P_{R_{k}} P_{N_{1}} \phi_{1}\right\|_{L^{2}}\left\|P_{N_{2}} \phi_{2}\right\|_{L^{2}}\left\|P_{N_{3}} \phi_{3}\right\|_{L^{2}},
\end{aligned}
$$

with $4<p<5$ and $q$ such that $2 / p+1 / q=1 / 2$, and $0<\varepsilon<1 / 2-2 / p$. Then (26) follows by summing up the squares with respect to $k$.

\section{Proof of the main Results}

Before we explain the proofs of Theorems 1.1 and 1.2 we summarize the results of the previous section in the following nonlinear estimate.

Proposition 4.1. Let $s \geq 1$ be fixed. Then, for all $0<T \leq 2 \pi$, and $u_{k} \in X^{s}([0, T)), k=1, \ldots, 5$, the estimate

$$
\left\|\mathcal{I}\left(\prod_{k=1}^{5} \widetilde{u}_{k}\right)\right\|_{X^{s}([0, T))} \lesssim \sum_{j=1}^{5}\left\|u_{j}\right\|_{X^{s}([0, T))} \prod_{\substack{k=1 \\ k \neq j}}^{5}\left\|u_{k}\right\|_{X^{1}([0, T))},
$$

holds true, where $\widetilde{u}_{k}$ denotes either $u_{k}$ or $\bar{u}_{k}$.

Proof. Let $I$ denote the interval $I=[0, T)$, and let $N \geq 1$ be given. Proposition 2.11 implies $\mathcal{I}\left(P_{\leq N} \prod_{k=1}^{5} \widetilde{u}_{k}\right) \in X^{s}(I)$ and

$$
\left\|\mathcal{I}\left(P_{\leq N} \prod_{k=1}^{5} \widetilde{u}_{k}\right)\right\|_{X^{s}(I)} \leq \sup _{\substack{v \in Y^{-s}(I): \\\|v\|_{Y^{-s}}=1}} \int_{0}^{2 \pi} \int_{\mathbb{T}^{3}} P_{\leq N} \prod_{k=1}^{5} \widetilde{u}_{k} \bar{v} d x d t
$$

We denote $u_{0}=P_{\leq N} v$. Then we need to prove the multilinear estimate

$$
\left|\int_{I \times \mathbb{T}^{3}} \prod_{k=0}^{5} \tilde{u}_{k} d x d t\right| \lesssim\left\|u_{0}\right\|_{Y^{-s}(I)} \sum_{j=1}^{5}\left(\left\|u_{j}\right\|_{X^{s}(I)} \prod_{\substack{k=1 \\ k \neq j}}^{5}\left\|u_{k}\right\|_{X^{1}(I)}\right)
$$

Once (28) is obtained the claimed estimate follows by letting $N \rightarrow \infty$. We consider extensions to $\mathbb{R}$ of $u_{k}$ which we will also denote with $u_{k}$ in 
the sequel of the proof, $k=0, \ldots, 5$, and (28) reduces to

$$
\left|\int_{I \times \mathbb{T}^{3}} \prod_{k=0}^{5} \tilde{u}_{k} d x d t\right| \lesssim\left\|u_{0}\right\|_{Y^{-s}} \sum_{j=1}^{5}\left(\left\|u_{j}\right\|_{X^{s}} \prod_{\substack{k=1 \\ k \neq j}}^{5}\left\|u_{k}\right\|_{X^{1}}\right) .
$$

We dyadically decompose

$$
\widetilde{u}_{k}=\sum_{N_{k} \geq 1} P_{N_{k}} \widetilde{u}_{k}
$$

In order for the integral in (29) to be nontrivial, the two highest frequencies must be comparable. Then, by the Cauchy-Schwarz inequality and symmetry it suffices to show that (with $L^{2}=L^{2}\left(I \times \mathbb{T}^{3}\right)$ )

$$
\begin{aligned}
S & =\sum_{\mathcal{N}}\left\|P_{N_{1}} \widetilde{u}_{1} P_{N_{3}} \widetilde{u}_{3} P_{N_{5}} \widetilde{u}_{5}\right\|_{L^{2}}\left\|P_{N_{0}} \widetilde{u}_{0} P_{N_{2}} \widetilde{u}_{2} P_{N_{4}} \widetilde{u}_{4}\right\|_{L^{2}} \\
& \lesssim\left\|u_{0}\right\|_{Y^{-s}} \sum_{j=1}^{5}\left\|u_{j}\right\|_{X^{s}} \prod_{\substack{k=1 \\
k \neq j}}^{5}\left\|u_{k}\right\|_{X^{1}},
\end{aligned}
$$

where $\mathcal{N}$ is as the set of all 6 -tuples $\left(N_{0}, N_{1}, \ldots, N_{5}\right)$ of dyadic numbers $N_{i} \geq 1$ satisfying

$$
N_{5} \leq \ldots \leq N_{1}, \quad \max \left\{N_{0}, N_{2}\right\} \approx N_{1} .
$$

We subdivide the sum into two parts $S=S_{1}+S_{2}$ :

Part $\mathcal{N}_{1}: N_{2} \leq N_{0} \approx N_{1}$ : Proposition 3.5 implies

$$
S_{1} \lesssim \sum_{\mathcal{N}_{1}} N_{2} N_{3} N_{4} N_{5}\left(\frac{N_{5}}{N_{1}}+\frac{1}{N_{3}}\right)^{\delta}\left(\frac{N_{4}}{N_{0}}+\frac{1}{N_{2}}\right)^{\delta} \prod_{k=0}^{5}\left\|P_{N_{k}} u_{k}\right\|_{Y^{0}}
$$

Using Cauchy-Schwarz, we easily sum up with respect to $N_{2}, N_{3}, N_{4}$ and $N_{5}$, and obtain

$$
S_{1} \lesssim \sum_{N_{0} \approx N_{1}}\left\|P_{N_{0}} u_{0}\right\|_{Y^{0}}\left\|P_{N_{1}} u_{1}\right\|_{Y^{0}} \prod_{k=2}^{5}\left\|u_{k}\right\|_{Y^{1}}
$$

Another application of Cauchy-Schwarz with respect to $N_{1}$ yields

$$
S_{1} \lesssim\left\|u_{0}\right\|_{Y^{-s}}\left\|u_{1}\right\|_{Y^{s}} \prod_{k=2}^{5}\left\|u_{k}\right\|_{Y^{1}}
$$

as needed.

Part $\mathcal{N}_{2}: N_{0} \leq N_{2} \approx N_{1}$. Proposition 3.5 implies

$$
S_{2} \lesssim \sum_{\mathcal{N}_{2}} N_{0} N_{3} N_{4} N_{5}\left(\frac{N_{5}}{N_{1}}+\frac{1}{N_{3}}\right)^{\delta} \prod_{k=0}^{5}\left\|P_{N_{k}} u_{k}\right\|_{Y^{0}}
$$


GWP OF THE ENERGY CRITICAL NLS WITH SMALL DATA IN $H^{1}\left(\mathbb{T}^{3}\right)$ 15

By Cauchy-Schwarz we sum with respect to $N_{3}, N_{4}$ and $N_{5}$ and obtain

$$
S_{2} \lesssim \sum_{N_{0} \leq N_{1} \approx N_{2}} N_{0}\left\|P_{N_{0}} u_{0}\right\|_{Y^{0}}\left\|P_{N_{1}} u_{1}\right\|_{Y^{0}}\left\|P_{N_{2}} u_{2}\right\|_{Y^{0}} \prod_{k=3}^{5}\left\|u_{k}\right\|_{Y^{1}} .
$$

For the remaining sum we apply directly Cauchy-Schwarz with respect to $N_{0}$ to obtain

$$
S_{2} \lesssim \sum_{N_{1} \approx N_{2}} N_{1}^{s+1}\left\|u_{0}\right\|_{Y^{-s}}\left\|P_{N_{1}} u_{1}\right\|_{Y^{0}}\left\|P_{N_{2}} u_{2}\right\|_{Y^{0}} \prod_{k=3}^{5}\left\|u_{k}\right\|_{Y^{1}} .
$$

Finally, we apply Cauchy-Schwarz with respect to $N_{1}$ to obtain

$$
S_{2} \lesssim\left\|u_{0}\right\|_{Y^{-s}}\left\|u_{1}\right\|_{Y^{s}} \prod_{k=2}^{5}\left\|u_{k}\right\|_{Y^{1}}
$$

The proof of the proposition is complete.

Proof of Theorem 1.1. We split the proof into several parts. The general strategy of the proof is well-known, see e.g. [7, 14]. We study the case $s=1$ in detail.

Part 1: Small data. From (27) we obtain

$$
\begin{aligned}
& \left\|\mathcal{I}\left(|u|^{4} u-|v|^{4} v\right)\right\|_{X^{1}([0, T))} \\
\leq & c\left(\|u\|_{X^{1}([0, T))}^{4}+\|v\|_{X^{1}([0, T))}^{4}\right)\|u-v\|_{X^{1}([0, T))},
\end{aligned}
$$

for all $0<T \leq 2 \pi$ and $u, v \in X^{1}([0, T))$.

For parameters $\varepsilon>0, \delta>0$, consider the sets

$$
\begin{aligned}
& B_{\varepsilon}:=\left\{\phi \in H^{1}\left(\mathbb{T}^{3}\right):\|\phi\|_{H^{1}} \leq \varepsilon\right\}, \\
& D_{\delta}:=\left\{u \in X^{1}([0,2 \pi)) \cap C\left([0,2 \pi) ; H^{1}\left(\mathbb{T}^{3}\right)\right):\|u\|_{X^{1}([0,2 \pi))} \leq \delta\right\} .
\end{aligned}
$$

The set $D_{\delta}$ is closed in $X^{1}([0,2 \pi))$, hence it is a complete space. With $L(\phi)=e^{i t \Delta} \phi$ and $N L(u)=-i \mathcal{I}\left(|u|^{4} u\right)$ we aim to solve the equation

$$
u=L(\phi)+N L(u)
$$

by the contraction mapping principle in $D_{\delta}$, for $\phi \in B_{\varepsilon}$. We have

$$
\|L(\phi)+N L(u)\|_{X^{1}([0,2 \pi))} \leq \varepsilon+c \delta^{5} \leq \delta,
$$

by choosing

$$
\delta=(4 c)^{-\frac{1}{4}} \text { and } \varepsilon=\delta / 2 .
$$

With the same choice it follows that

$$
\|N L(u)-N L(v)\|_{X^{1}([0,2 \pi))} \leq \frac{1}{2}\|u-v\|_{X^{1}([0,2 \pi))}
$$


which shows that the nonlinear map $L(\phi)+N L(u)$ has a unique fixed point in $D_{\delta}$ (Concerning uniqueness in the full space, see below). Similarly, for $\phi, \psi \in B_{\varepsilon}$ and the corresponding fixed points $u, v \in D_{\delta}$ it follows

$$
\|u-v\|_{X^{1}([0, T))} \leq\|\phi-\psi\|_{H^{1}}+2 c \delta^{4}\|u-v\|_{X^{1}([0, T))},
$$

which yields the Lipschitz dependence.

Part 2: Large data. Let $r>0$ and $N \geq 1$ be given. For parameters $\varepsilon, \delta, R, T$, with $0<\varepsilon \leq r, 0<\delta \leq R$, consider the sets

$$
\begin{aligned}
B_{\varepsilon, r} & :=\left\{\phi \in H^{1}\left(\mathbb{T}^{3}\right):\left\|\phi_{>N}\right\|_{H^{1}} \leq \varepsilon,\|\phi\|_{H^{1}} \leq r\right\}, \\
D_{\delta, R, T}:=\left\{u \in X^{1}([0, T)) \cap C\left([0, T) ; H^{1}\left(\mathbb{T}^{3}\right)\right):\right. & =\left\{u_{>N}\left\|_{X^{1}([0, T))} \leq \delta,\right\| u \|_{X^{1}([0, T))} \leq R\right\},
\end{aligned}
$$

where $f_{>N}=\left(I-P_{\leq N}\right) f$. For $\phi \in B_{\varepsilon, r}$ we have

$$
\left\|[L(\phi)+N L(u)]_{>N}\right\|_{X^{1}([0, T))} \leq \varepsilon+\left\|[N L(u)]_{>N}\right\|_{X^{1}([0, T))} .
$$

We split $N L(u)=N L_{1}\left(u_{\leq N}, u_{>N}\right)+N L_{2}\left(u_{\leq N}, u_{>N}\right)$, such that $N L_{1}$ is at least quadratic in $u_{>N}$ and $N L_{2}$ is at least quartic in $u_{\leq N}$. In view of (27) we obtain

$$
\left\|N L_{1}\left(u_{\leq N}, u_{>N}\right)\right\|_{X^{1}([0, T))} \leq c \delta^{2} R^{3} .
$$

Concerning the estimate for $N L_{2}$ we recall (see Remark 4) that it suffices to control the nonlinearity in $L^{1}\left([0, T) ; H^{1}\right)$. The latter follows from a Sobolev embedding type argument

$$
\begin{aligned}
& \left\|N L_{2}\left(u_{\leq N}, u_{>N}\right)\right\|_{X^{1}([0, T))} \leq c_{1}\|u\|_{L^{\infty}\left([0, T) ; H^{1}\right)}\left\|u_{\leq N}\right\|_{L^{4}\left([0, T) ; L^{\infty}\right)}^{4} \\
& +c_{1} N\|u\|_{L^{\infty}\left([0, T) ; L^{6}\right)}\left\|u_{\leq N}\right\|_{L^{4}\left([0, T) ; L^{12}\right)}^{4} \leq c_{2} N^{2} T R^{5} .
\end{aligned}
$$

Similarly to (34), one can show that

$$
\left\|N L_{1}\left(u_{\leq N}, u_{>N}\right)-N L_{1}\left(v_{\leq N}, v_{>N}\right)\right\|_{X^{1}([0, T))} \leq c_{3} \delta R^{3}\|u-v\|_{X^{1}([0, T))} .
$$

Moreover, as in (35), it follows

$\left\|N L_{2}\left(u_{\leq N}, u_{>N}\right)-N L_{2}\left(v_{\leq N}, v_{>N}\right)\right\|_{X^{1}([0, T))} \leq c_{4} N^{2} R^{4} T\|u-v\|_{X^{1}([0, T))}$.

Let $C \geq 1$ be the maximum of $c, c_{1}, \ldots, c_{4}$. By choosing the parameters

$$
R:=4 r, \delta:=\left(8 C R^{3}\right)^{-1}, \varepsilon:=\delta / 2, T:=\delta\left(8 C R^{5} N^{2}\right)^{-1},
$$

we have found that for any $\phi \in B_{\varepsilon, r}$ the map

$$
L(\phi)+N L(u): D_{\delta, R, T} \rightarrow D_{\delta, R, T}
$$

is a strict contraction, which has a unique fixed point $u$ and $\phi \mapsto u$ is Lipschitz continuous with constant 2 . 
Part 3: Conclusion. Concerning uniqueness, we note that by translation invariance in $t$ it suffices to consider

$$
u, v \in X^{1}([0, T)) \cap C\left([0, T) ; H^{1}\left(\mathbb{T}^{3}\right)\right) \text { such that } u(0)=v(0),
$$

and show $u=v$ for arbitrarily small $T>0$. Indeed, this follows from the uniqueness of the fixed point in balls of arbitrary radius already proved in Part 2.

Now, let $\phi_{*} \in H^{1}\left(\mathbb{T}^{3}\right)$ and $\varepsilon>0$ be as in (36) for given $r=2\left\|\phi_{*}\right\|_{H^{1}}$ (notice that this choice is independent of $N$ ). Choose $N \geq 1$ large enough such that $\left\|\left[\phi_{*}\right]_{>N}\right\|_{H^{1}} \leq \varepsilon / 2$. Then, for all $\phi \in B_{\varepsilon / 2}\left(\phi_{*}\right)$ we have $\phi \in B_{\varepsilon, r}$, and by Part 2 we find $T=T(r, N)>0$ and a unique solution $u \in X^{1}([0, T)) \cap C\left([0, T) ; H^{1}\left(\mathbb{T}^{3}\right)\right)$ which depends Lipschitz continuously on the initial data $\phi$.

Finally, we remark that by the tame estimate (27) we can easily prove persistence of higher order Sobolev regularity, with a (conceivably shorter) lifespan depending on $\|\phi\|_{H^{s}}$. However, an a posteriori iteration argument shows that the time of existence must be at least $T\left(\phi_{*}\right)$.

Proof of Theorem 1.2. By part 1 and 3 of the previous proof it suffices to provide a suitable a priori bound on the solution in $H^{1}$.

In the defocusing case, this obviously follows from conservation laws (5) and (6) the Sobolev embedding $H^{1}\left(\mathbb{T}^{3}\right) \hookrightarrow L^{6}\left(\mathbb{T}^{3}\right)$ :

$$
\|u(t)\|_{L^{2}}^{2}+\|\nabla u\|_{L^{2}}^{2} \leq 2 E(u(0))+2 M(u(0)) \leq\|u(0)\|_{H^{1}}^{2}+d^{2}\|u(0)\|_{H^{1}}^{6} .
$$

If $\|u(0)\|_{H^{1}}$ is chosen to be small enough, it follows that for $\varepsilon$ as in (32) the solution satisfies $\|u(t)\|_{H^{1}} \leq \varepsilon$ on any interval of existence, and we can iterate the argument from Part 1 indefinitely.

In the focusing case, we combine the conservation laws (5) and (6) with a continuity argument, based on the inequality

$$
\begin{aligned}
\|u(t)\|_{H^{1}}^{2} & =2 E(u(0))+2 M(u(0))+\frac{1}{3}\|u(t)\|_{L^{6}}^{6} \\
& \leq\|u(0)\|_{H^{1}}^{2}+d^{2}\|u(0)\|_{H^{1}}^{6}+d^{2}\|u(t)\|_{H^{1}}^{6},
\end{aligned}
$$

which is valid for any solution $u \in X^{1}([0,2 \pi))$.

We consider $f(x)=x-d^{2} x^{3}$ for $x \geq 0$, which increases from 0 to its maximum value $2(3 \sqrt{3} d)^{-1}$ and satisfies $f(x) \geq \frac{2}{3} x$ on the interval $I=\left[0,(\sqrt{3} d)^{-1}\right]$. We have shown above that $f\left(\|u(t)\|_{H^{1}}^{2}\right)<\varepsilon_{0}^{2}$, for all $t \in[0,2 \pi)$ and all initial data satisfying

$$
\|u(0)\|_{H^{1}}^{2}+d^{2}\|u(0)\|_{H^{1}}^{6}<\varepsilon_{0}^{2} .
$$

By choosing $\varepsilon_{0}^{2}=\min \left\{2(3 \sqrt{3} d)^{-1}, 2 / 3 \varepsilon^{2}\right\}$, with $\varepsilon$ as in (32), the continuity of $t \mapsto\|u(t)\|_{H^{1}}^{2}$ implies that $\|u(t)\|_{H^{1}}^{2} \in I$ for all $t \in[0,2 \pi)$, and 
therefore $\|u(t)\|_{H^{1}}^{2} \leq 3 / 2 \varepsilon_{0}^{2} \leq \varepsilon^{2}$ for all $t \in[0,2 \pi)$, which allows us to iterate the small data local well-posedness argument.

\section{REFERENCES}

1. Jean Bourgain, Fourier transform restriction phenomena for certain lattice subsets and applications to nonlinear evolution equations. I. Schrödinger equations, Geom. Funct. Anal. 3 (1993), no. 2, 107-156. MR1209299 (95d:35160a)

2. $\ldots$, On Strichartz's inequalities and the nonlinear Schrödinger equation on irrational tori, Mathematical aspects of nonlinear dispersive equations, Ann. of Math. Stud., vol. 163, Princeton Univ. Press, Princeton, NJ, 2007, pp. 1-20. MR2331676 (2008j:35165)

3. Nicolas Burq, Patrick Gérard, and Nikolay Tzvetkov, Strichartz inequalities and the nonlinear Schrödinger equation on compact manifolds, Amer. J. Math. 126 (2004), no. 3, 569-605. MR2058384 (2005h:58036)

4. B Bilinear eigenfunction estimates and the nonlinear Schrödinger equation on surfaces, Invent. Math. 159 (2005), no. 1, 187-223. MR2142336 (2005m:35275)

5. __ Multilinear eigenfunction estimates and global existence for the three dimensional nonlinear Schrödinger equations, Ann. Sci. École Norm. Sup. (4) 38 (2005), no. 2, 255-301. MR2144988 (2006m:35337)

6. - Global solutions for the nonlinear Schrödinger equation on threedimensional compact manifolds, Mathematical aspects of nonlinear dispersive equations, Ann. of Math. Stud., vol. 163, Princeton Univ. Press, Princeton, NJ, 2007, pp. 111-129. MR2333209 (2008i:58026)

7. Thierry Cazenave and Fred B. Weissler, The Cauchy problem for the critical nonlinear Schrödinger equation in $H^{s}$, Nonlinear Anal. 14 (1990), no. 10, 807836. MR1055532 (91j:35252)

8. James Colliander, Markus Keel, Gigliola Staffilani, Hideo Takaoka, and Terence C. Tao, Global well-posedness and scattering for the energy-critical nonlinear Schrödinger equation in $\mathbb{R}^{3}$, Ann. of Math. (2) 167 (2008), no. 3, 767-865. MR2415387 (2009f:35315)

9. Martin Hadac, Sebastian Herr, and Herbert Koch, Well-posedness and scattering for the KP-II equation in a critical space, Ann. Inst. $\mathrm{H}$. Poincaré - AN 26 (2009), no. 3, 917-941, Erratum published at http://dx.doi.org/10.1016/j.anihpc.2010.01.006. MR2526409

10. Sebastian Herr, Daniel Tataru, and Nikolay Tzvetkov, Strichartz estimates for partially periodic solutions to Schrödinger equations in $4 d$ and applications, Preprint, arXiv:1011.0591, 2010.

11. Markus Keel and Terence Tao, Endpoint Strichartz estimates, Amer. J. Math. 120 (1998), no. 5, 955-980. MR1646048 (2000d:35018)

12. Herbert Koch and Daniel Tataru, Dispersive estimates for principally normal pseudodifferential operators, Comm. Pure Appl. Math. 58 (2005), no. 2, 217284. MR2094851 (2005m:35323)

13. A A priori bounds for the $1 D$ cubic NLS in negative Sobolev spaces, Int. Math. Res. Not. IMRN 2007 (2007), no. 16, Art. ID rnm053, 36p. MR2353092 (2010d:35307) 
GWP OF THE ENERGY CRITICAL NLS WITH SMALL DATA IN $H^{1}\left(\mathbb{T}^{3}\right) \quad 19$

14. Terence Tao, Scattering for the quartic generalised Korteweg-de Vries equation, J. Differential Equations 232 (2007), no. 2, 623-651. MR2286393 (2008i:35178)

Mathematisches Institut, Universität Bonn, Endenicher Allee 60, 53115 BONn, GERMANY

E-mail address: herr@math.uni-bonn.de

Department of Mathematics, University of California, Berkeley, CA 94720-3840, USA

E-mail address: tataru@math.berkeley.edu

Département de Mathématiques, Université de Cergy-Pontoise, 2, avenue Adolphe Chauvin, 95302 Cergy-Pontoise Cedex, France and Institut Universitaire De France

E-mail address: nikolay.tzvetkov@u-cergy.fr 